\title{
Caractérisation des apports sédimentaires et morphologie du lac du barrage hydroélectrique d'Ayame 1 (Sud-Est Côte d'Ivoire)
}

\author{
N'diaye Hermann MELEDJE $^{1 *}$, Kouakou Lazare KOUASSI ${ }^{1}$, Yao Alexis N'GO ${ }^{2}$, \\ Kan Martin KOUASSI ${ }^{1}$, Issiaka SAVANE ${ }^{2}$ et Kouamé AKA ${ }^{1}$ \\ ${ }^{1}$ Centre de Recherche en Ecologie, Laboratoire de Géologie Marine, Sédimentologie et Environnement, 08 \\ BP 109 Abidjan 08, Côte d'Ivoire. \\ ${ }^{2}$ Université Nangui Abrogoua, Laboratoire Géosciences et Environnement, 01 BP 10588 Abidjan 01, \\ Côte d'Ivoire. \\ *Auteur correspondant, E-mail: meledjendiay@yahoo.fr
}

\section{RESUME}

Une analyse sédimentologique réalisée entre février 2004 et octobre 2013 a permis d'évaluer les charges solides en suspension et de caractériser les sédiments du lac du barrage d'Ayamé. La concentration moyenne en matières en suspension $\left(6,15 \mathrm{mg} . \mathrm{L}^{-1}\right)$ et la turbidité moyenne $(17,5 \mathrm{NTU})$ montrent que les eaux du lac sont relativement peu chargées. Pour les 34 sédiments étudiés, une carte de Kohonen (SOM) des réseaux de neurone a permis de regrouper les sédiments en 3 classes ayant les caractéristiques semblables à partir de leur diamètre. Cette classification constitue une première approche de regroupement des sédiments basée sur les diamètres des particules à l'aide des cartes de Kohonen. Le groupe I est caractérisé par un faible taux $(5,532 \%)$ de sédiments de diamètre $5000 \mu \mathrm{m}$. Le groupe II renferme majoritairement des sédiments grossiers $(84,75 \%)$. Le troisième groupe se caractérise par des sédiments grossiers $(65,34 \%)$ à moyen $(16,27 \%)$. Les résultats obtenus par l'application d'un modèle débits liquides-débits solides saisonniers sur le lac d'Ayamé sont très encourageants car les coefficients de corrélation varient de 0,64 à 0,91 . Par ailleurs, cette étude a permis de réaliser la première carte bathymétrique du lac d'Ayamé 1, 54 ans après sa mise en eau. (c) 2014 International Formulae Group. All rights reserved.

Mots clés: Flux solide, carte de Kohonen, réseaux de neurone, bathymétrie, lac d'Ayamé 1.

\section{INTRODUCTION}

Les retenues d'eau de la Côte d'Ivoire sont de plus en plus confrontées aux problèmes hydro sédimentaires. Des travaux récents (Ouattara, 2000; Yapo 2002; Kouassi, 2007; Kouamé et al., 2010 ; Kouamé et al., 2011 ; Groga, 2012 ; Kouassi et al., 2013) ont montré que ces retenues d'eau, qui représentent des atouts majeurs pour soutenir le développement du pays, sont menacées par les variations hydrologiques, la pollution et les problèmes d'envasement. Le comblement des retenues d'eau est dû aux activités agricoles, l'urbanisation rapide et incontrôlée qui intensifient les phénomènes d'érosion et augmentent la production de sédiments (N'Go, 2000 ; Grismer, 2012).

L'envasement et l'eutrophisation des réservoirs provoquent des inondations, des modifications significatives des paramètres physico-chimiques et un développement rapide des plantes aquatiques. Malgré les 
dangers inhérents au comblement des retenues d'eau avec le temps, aucune étude sédimentologique et morphologique n'a encore été entreprise sur le lac du barrage hydroélectrique d'Ayamé 1, le plus vieux barrage hydroélectrique de la Côte d'Ivoire mis en eau en 1959. Or, Bessenassé (2003) et Okacha et al. (2002) soulignent que l'alluvionnement des retenues d'eau conduisant à la réduction de leur durée de vie constitue une des conséquences les plus néfastes de l'érosion hydrique. La gestion des écosystèmes lacustres nécessite une connaissance approfondie du fonctionnement hydrodynamique et sédimentaire afin de proposer des scénarios d'aménagements adaptés aux différentes contraintes du milieu : maintien de la qualité de l'eau, amélioration de l'habitat des poissons et des organismes aquatiques, protection des ouvrages, développement des activités récréatives et écotouristiques (Zimmerman et al., 2003 ; Lenhart et al., 2010 ; Ryan et al., 2014).

Cet article présente la première carte bathymétrique du lac d'Ayamé 1, 54 ans après sa mise en eau et se propose de modéliser les flux des matières en suspension dans le lac d'Ayamé 1. Il présente également les caractéristiques granulométriques des sédiments et leur répartition spatiale dans le lac d'Ayamé 1.

\section{MATERIEL ET METHODES \\ Présentation de la zone d'étude}

Le bassin de la Bia est un bassin versant transfrontalier entre la Côte d'Ivoire et le Ghana (Figure 1). Ce bassin a une superficie d'environ $1500 \mathrm{~km}^{2}$. La rivière Bia abrite les lacs des barrages hydroélectriques d'Ayamé 1 et 2, les plus vieux barrages de la Côte d'Ivoire, construits respectivement en 1959 et 1965 .

Le bassin de la Bia appartient au climat équatorial humide, caractérisé par l'importance de la première saison de pluies (mars à juillet) avec un maximum de précipitation en juin et une deuxième saison humide assez forte de septembre à novembre (Durand et Guiral, 1994) (Figure 2). Selon les travaux de Adjanohoun et Guillaumet (1971) et de Girard et al. (1970), la rivière Bia s'écoulait entièrement sous forêt dense avec un débit moyen de $82 \mathrm{~m}^{3} / \mathrm{s}$.

\section{Echantillonnage des sédiments et d'eau}

Les sédiments du lac d'Ayamé 1 ont été prélevés à l'aide d'une benne Van Veen tandis que les échantillons d'eau ont été recueillis au moyen d'une bouteille hydrologique. Pour réaliser cette étude, 102 échantillons de sédiments et 33 échantillons d'eau ont été prélevés sur 12 stations dans le lac (Figure 3). Les positions des stations de prélèvement ont été déterminées à l'aide d'un GPS. L'échantillonnage a été réalisé pendant la petite et la grande saison sèche (février et août 2004, février et août 2012, février et août 2013) et pendant la petite et la grande saison de pluie (juin et octobre 2004, juin et octobre 2012, juin et octobre 2013).

La conductivité, la température, le $\mathrm{pH}$, l'oxygène dissous et la turbidité ont été mesurés in-situ.

\section{Bathymétrie du lac d'Ayamé 1}

L'étude de la morphologie du fond du lac d'Ayamé 1 a été effectuée par l'intermédiaire de levés bathymétriques, réalisés à l'aide d'un échosondeur de type Lowrance, modèle LMS-160. Les cartes sédimentologiques et les cartes de positionnement ont été établies à l'aide du logiciel ARCGIS 10.

\section{Caractérisation des sédiments du lac d'Ayamé 1 \\ Analyses granulométriques}

Les sédiments ont été analysés selon la technique décrite par Saaidi (1991). Les matières organiques et les débris coquilliers ont été éliminés respectivement à l'aide de l'eau oxygénée $30 \%$ et de l'acide chlorhydrique $50 \%$. Après élimination de la fraction inférieure à $63 \mu \mathrm{m}$, chaque échantillon de sable a subi une analyse granulométrique à sec sur une colonne de 16 tamis (série AFNOR). Les sables du lac d'Ayamé 1 ont été caractérisés à travers la 
moyenne granulométrique $(\mathrm{Mz})$, le skewness (Sk) et l'écart type (So), déterminés à l'aide des méthodes de Folk et Word (1957).

\section{Classification des sédiments}

La plupart des échantillons de sables prélevés en pleine eau sont argilo-vaseux (soit 88 échantillons sur 102) et n'ont pu être analysés par densimétrie. Pour les 34 sédiments étudiés, une carte auto organisatrice de Kohonen (SOM) des réseaux de neurone a été établie. L'analyse de SOM a été utilisée pour établir une liaison entre les unités d'entrée, représentées par les moyennes granulométriques et les unités de sortie associées pour les simplifier et caractériser les sédiments; ceci pour obtenir une représentation visuelle des variations spatiales. Une fois que la carte organisatrice est établie, il peut être difficile de distinguer les frontières entre différents sous ensembles. La classification hiérarchique est alors utilisée pour créer une représentation sous forme d'arbres ou de dendrogrammes qui donnent les nombres de répartitions par classe existant dans la population étudiée (Bamory et al., 2010). Ceci permet de grouper les unités de la carte en différents sous ensembles selon le vecteur poids des neurones de sortie. Les SOM permettent de ressortir des groupes semblables à partir des matrices des données complexes (Kohonen, 2001).

\section{Caractérisation physicochimique des eaux du lac d'Ayamé 1 \\ Evaluation des charges solides en suspension} Les matières en suspension sont obtenues suivant la norme Afnor : T 90-119. Les échantillons d'eau ont été filtrés sur une rampe de filtration millipore à l'aide de filtres WHATMAN GF/F circulaires de $47 \mathrm{~mm}$ de diamètre et de porosité $0,45 \mu \mathrm{m}$ préalablement pesés. Après séchage à $105{ }^{\circ} \mathrm{C}$, les filtres ont été soigneusement récupérés et repesés afin de déterminer les concentrations totales de matières en suspension exprimées en mg/L (Bouanani, 2004). La turbidité a été mesurée à l'aide d'un turbidimètre de marque HANNA INSTRUMENTS, modèle LP 2000. Un flacon de $10 \mathrm{~mL}$ rempli d'échantillon d'eau et placé dans le turbidimètre permet une lecture directe des valeurs de la turbidité exprimées en NTU.

Le calcul du débit solide en suspension est fonction du débit liquide observé (valeurs instantanées $\mathrm{Q}$ en $\mathrm{m}^{3} / \mathrm{s}$ ) et de la concentration de matières en suspension (Bouanani, 2004 ; Kouassi et al., 2013) :

$T_{S S}=C_{M E S} \cdot Q \cdot k$

Avec $T_{S S}=$ Flux des matières en suspension $(\mathrm{t} / \mathrm{j}) ; C_{M E S}=$ concentration des MES (mg/l) ; $Q=$ débit liquide $\left(\mathrm{m}^{3} / \mathrm{s}\right) ; \mathrm{k}=$ unité de conversion (0,0864 pour Tss en tonne/jour). Les apports solides peuvent être quantifiés annuellement, mensuellement ou saisonnièrement.

\section{Corrélation entre les différents paramètres physicochimiques}

Cette étude repose sur une analyse statistique corrélative (corrmap) développée sur matlab. Le corrmap.m fonction a été utilisé pour étudier l'évolution des paramètres physicochimiques en fonction de la profondeur et afficher une carte de la matrice de corrélation pseudo en réarrangeant les variables de sorte qu'ils soient regroupés et corrélées entre elles. Un algorithme des plus proches k-voisins modifiés, est utilisé pour changer l'ordre des variables. Les variables ont été disposées de sorte que celles qui sont très corrélées, soient proches les unes des autres. La figure produit une carte qui permet de visualiser la proximité entre les différentes variables.

\section{RESULTATS}

Nature et répartition spatiale des sédiments

Les sédiments du lac d'Ayamé 1 sont dominés par les vases (85\%). Ces vases se rencontrent sur toute l'étendue du fond du lac. Les argiles sableuses se rencontrent en bordure des îles. Le faciès sableux et les graviers se trouvent sur les berges (Figure 4).

\section{Morphologie du fond du lac}

La Figure 5 présente la carte bathymétrique du lac d'Ayamé 1. Cette carte a été réalisée à l'aide des levés bathymétriques effectués en juin 2005. La cote 89,36 m du 
plan d'eau a été retenue comme cote de référence. L'examen détaillé de la bathymétrie du lac d'Ayamé 1 fait apparaître une morphologie de fond relativement complexe. On constate que le fond du lac présente un relief un peu plus accidenté. Sur cette carte bathymétrique, il y a un principal chenal d'écoulement de direction Sud Est débouchant sur la prise d'eau. On remarque que les profondeurs augmentent progressivement de l'amont vers la digue. Aux voisinages de la centrale, notamment au droit de la prise d'eau où transitent les débits turbinés, les profondeurs sont assez importantes et atteignent $34 \mathrm{~m}$.

\section{Analyse granulométrique des sédiments}

Les courbes cumulatives semilogarithmiques des sédiments du lac en général ont la forme d'un $\mathbf{S}$ à forte pente, bien redressé et bien régulier malgré l'existence de légères différences dans leurs allures (Figure 6). Ceci indique qu'il s'agit de sables bien classés dans un milieu peu agité avec évacuation des particules fines vers le fond par les courants. Une telle forme des courbes montre aussi qu'on a un stock sédimentaire homogène et des conditions d'énergie adaptées à la charge transportée.

Les courbes cumulatives des échantillons $\mathrm{S}_{\mathrm{O}}$ et carotte cote magasin, prélevés de la plage, ont la forme d'un S plus ou moins étalé qui indique des sables à classement moyen et un milieu plus ou moins agité. Les courbes cumulatives des échantillons en amont du lac ont, généralement, la forme d'un $\mathrm{S}$ plus ou moins régulier (Figure 6). Ceci témoigne d'un bon classement des sédiments et d'un stock de sédiment homogène. Les valeurs de la moyenne de tous les échantillons sont comprises entre $286 \mu \mathrm{m}$ et $2680 \mu \mathrm{m}$, ce qui indique des sables grossiers à moyens (Tableau 1). La taille des grains de sables diminue en allant des petits fonds de faibles profondeurs (2 m) à d'autres plus profonds $(10 \mathrm{~m})$. Au niveau de la station S0 et carotte coté magasin sont des sables très grossiers.
Le skewness des sédiments superficiels varie de $-0,02$ à 0,2 (Tableau 1). Les courbes granulométriques présentent une asymétrie vers les grossiers au niveau des petits fonds peu profonds $(2 \mathrm{~m})$ et tendent à devenir asymétriques et très asymétriques vers les fins en allant vers les petits fonds les plus profonds $(10 \mathrm{~m})$. Ceci témoigne l'existence du tri granulométrique, déjà constaté, en allant des berges peu profondes vers le chenal (Figure 5).

Le coefficient d'uniformité est inférieur à 2 pour tous les échantillons. Donc, les sables présentent une granulométrie uniforme. Comme l'illustre la Figure 4, dans les lithofaciès du système lacustre, dominent six types, à savoir : sable, sable vaseux, sable très vaseux, vase, vase sableuse et vase très sableuse. Ces faciès sont issus de l'altération et de la désagrégation de la roche-mère et des sols du bassin versant.

\section{Classification des sédiments du lac d'Ayamé}

Le Tableau 1 montre que seulement 8 échantillons sont renseignés soit environ $77 \%$ de perte d'information sur la caractérisation des sédiments du lac d'Ayamé. Il s'agit de rentabiliser les informations perdues. La classification permet de repartir les 34 échantillons en 3 groupes avec une perte d'information de $8 \%$. La distribution granulométrique des groupes est présentée à la Figure 8 , celles des échantillons par groupe à la Figure 9. Ainsi, en choisissant la distance euclidienne de 1 , on obtient trois groupes d'échantillons qui sont représentés à la Figure 8.

L'algorithme de la carte de Kohonen a permis en premier lieu de regrouper les 34 sédiments dans les 20 cellules de la carte autoorganisatrice de Kohonen. La classification numérique par la méthode Ward nous a permis de grouper les sédiments par rapport à la moyenne granulométrique. Il ressort donc, du dendrogramme de classification, trois groupes distincts (Figure 7). Le premier est représenté par les sédiments $\mathrm{S} 6, \mathrm{~S} 7, \mathrm{~S} 12, \mathrm{~S} 13$, S17, S19, S21, S22, S24, S27, S28, S30, S31, $\mathrm{S} 32$, S31. Alors que le second regroupe les S1, S8, S9, S14, S15, S16, S18, S20, S23, 
S25, S26. Les cartes de distribution (« distribution maps » ou « component planes »), issues de la carte de Kohonen, sont représentées à la Figure 9. Elles permettent de visualiser la répartition des sédiments en fonction de leur diamètre granulométrique.

Les cellules foncées en rouge représentent des valeurs élevées, alors que les cellules bleues représentent des valeurs faibles. L'échelle $\mathrm{d}$ indique les valeurs transformées de moyenne granulométrique par le logarithme. Une carte de 20 cellules (6 lignes X 5 colonnes) avec des erreurs de quantification de 0,333 et de topographie de 0,000 a été retenue pour cette analyse. Cette répartition permet, pour chaque diamètre granulométrique, de discriminer les différents groupes de sédiments obtenus. L'analyse de la répartition sur la carte de Kohonen des valeurs des différents diamètres granulométriques permet de faire la différenciation suivante des trois groupes de sédiments étudiés : le groupe I est constitué moyennement des sédiments grossiers $(51,82 \%)$. Ce groupe est caractérisé par un faible taux $5,532 \%$ ) de sédiments de diamètre $5000 \mu \mathrm{m}$ (Tableau 2). Les sédiments appartenant à ce groupe ont un faible taux d'éléments grossiers (de 5000 $\mu \mathrm{m}$ à $1200 \mu \mathrm{m}$ ), remarquable par la couleur de leurs cellules dominée par le bleu. Le gradient des valeurs indique que le groupe II renferme majoritairement des sédiments grossiers (84,75\%). Les sédiments de ce groupe sont les plus riches en éléments grossiers et fins (Tableau 2). Le troisième groupe se caractérise par des sédiments grossiers $(65,34 \%)$ à moyen $(16,27 \%)$.

\section{Corrélation entre les paramètres physicochimiques}

La matrice de corrélation appliquée sur les variables physicochimiques en fonction des saisons et de la profondeur montre qu'il existe une bonne corrélation entre l'ensemble des variables (Figure 10). Sur la matrice, les plus fortes valeurs sont observées sur la diagonale et la corrélation entre les différents points décroît ensuite avec la distance entre ces points. On peut cependant remarquer que la corrélation entre les points, même éloignés les uns des autres, reste relativement forte.

Il existe une très forte corrélation positive entre la concentration en matières en suspension et la turbidité $(\mathrm{r}=0,92)$. La turbidité de l'eau est influencée par les charges solides en suspension. Par ailleurs, le $\mathrm{pH}$, l'oxygène dissous et la température présentent une corrélation positive en fonction de la profondeur alors que la conductivité, la potentialité, la turbidité et les MES présentent une corrélation négative.

\section{Evolution de la turbidité et des MES}

Les variations saisonnières des MES et de la turbidité montrent que pendant les saisons sèches, les eaux du lac d'Ayamé 1 sont faiblement chargées. Ce qui se traduit par une faible concentration en MES (4,6 mg/L) et une faible turbidité (14,63 NTU). En revanche, pendant les saisons des pluies, les MES (7,7 mg/L) et la turbidité (21,12 NTU) deviennent relativement importantes.

En saison sèche, la turbidité diminue de 18 NTU à 12 NTU entre $0,5 \mathrm{~m}$ et $2 \mathrm{~m}$. Audelà de $2 \mathrm{~m}$, elle augmente progressivement avec la profondeur jusqu'à atteindre 19 NTU à $15 \mathrm{~m}$. Les valeurs de MES oscillent entre 5,40 et $6,02 \mathrm{mg} / \mathrm{L}$ en saison sèche et entre 4,55 et $9,45 \mathrm{mg} / \mathrm{L}$ en saison pluvieuse (Figure 11).

\section{Relations entre débits solides et débits liquides}

L'étude porte sur les valeurs instantanées des débits liquides et des débits solides (Figure 12). Pour les données de la station étudiée, les regroupements ont été effectués suivant quatre saisons: petite saison sèche (août), petite saison pluvieuse (octobre), grande saison sèche (février) et grande saison pluvieuse (juin). Les graphes de la Figure 12 illustrent ces relations pour les données saisonnières.

Les corrélations obtenues sont toutes significatives et varient dans l'intervalle $(0,64-0,91)$, ce qui permet de conclure que ces régressions ont donné des résultats acceptables. La relation liant les débits liquides aux débits solides en suspension est de type puissance de forme générale 
$Q_{s}=a Q_{l}^{b}$. Le Tableau 3 résume les résultats obtenus. Dans la période d'étude, les crues les plus importantes et les mieux suivies ont été sélectionnées (nombre important de prélèvements durant l'événement) et utilisées dans l'estimation.

\section{Evaluation du flux des sédiments}

Les flux particulaires en suspension évalués sont plus élevés en saison pluvieuse $(58,20 \mathrm{t} / \mathrm{j})$ qu'en saison sèche $(11,41 \mathrm{t} / \mathrm{j})$ à
Ayamé 1 (Tableau 4). Les variations des pluies, des apports liquides et des flux particulaires mettent en évidence une étroite corrélation entre les saisons hydrologiques et les concentrations en MES dans le lac d'Ayamé 1. En effet, pendant la saison pluvieuse, les eaux de ruissellement transportent plus de MES dans le plan d'eau que pendant la saison sèche.

Tableau 1 : Caractéristiques granulométriques des sédiments.

\begin{tabular}{|c|c|c|c|c|c|c|c|c|}
\hline Echantillon & $\Phi 16$ & Ф50 & \$84 & $\Phi 95$ & Ф5 & Moy. $(\mu \mathrm{m})$ & écart-type & skewness \\
\hline $\mathbf{S}_{\mathbf{0}}$ & - & - & 2,22 & 2,84 & - & 286,67 & - & - \\
\hline $\mathbf{S}_{1}$ & - & 0,42 & 0,26 & 1,89 & - & 1305 & - & - \\
\hline $\mathbf{S}_{2}$ & $-2,32$ & $-2,17$ & 1,56 & 2,43 & - & 2896,67 & - & - \\
\hline $\mathbf{S}_{\mathbf{3}}$ & $-2,32$ & 0,23 & 1,84 & 2,46 & - & 2260 & - & - \\
\hline $\mathbf{S}_{4}$ & $-2,17$ & 0,51 & 1,54 & 2,32 & - & 2115 & - & - \\
\hline$S_{5}$ & $-2,32$ & - & 1,18 & 2,4 & - & 2680 & - & - \\
\hline$S_{6}$ & 0,01 & 0,12 & 2,79 & 3,06 & $-1,58$ & 486,67 & 1,4 & 0,24 \\
\hline $\mathbf{S}_{7}$ & 0 & - & 2,74 & 3,43 & $-1,85$ & 503 & 1,48 & - \\
\hline $\mathbf{S}_{8}$ & - & $-2,29$ & 0,74 & 2,25 & - & 1600 & - & - \\
\hline $\mathbf{S}_{9}$ & - & 0,57 & 0,74 & 2,22 & - & 1500 & - & - \\
\hline$S_{10}$ & $-2,26$ & 3,47 & 2 & 2,74 & - & 1916,67 & - & - \\
\hline$S_{11}$ & $-1,79$ & $-1,29$ & 1,32 & 1,64 & $-2,56$ & 1750 & 1,41 & 0,07 \\
\hline$S_{12}$ & $-1,07$ & 0,4 & 2,51 & 3,18 & $-2,23$ & 1008,33 & 1,72 & 0,07 \\
\hline$S_{13}$ & $-0,63$ & 0,32 & 2,56 & 3,18 & $-2,14$ & 823,33 & 1,6 & 0,14 \\
\hline$S_{14}$ & - & 0,62 & $-0,32$ & 1,86 & - & 416,67 & - & - \\
\hline$S_{15}$ & - & 0,51 & 0,69 & 2,25 & - & 1873,33 & - & - \\
\hline$S_{16}$ & - & - & $-2,81$ & 2 & - & 2333,33 & - & - \\
\hline$S_{17}$ & $-1,14$ & - & 2,63 & 3,32 & - & 3120,67 & - & - \\
\hline$S_{18}$ & - & - & 0 & 2,56 & - & 333,33 & - & - \\
\hline$S_{19}$ & $-0,38$ & 11,23 & 2,62 & 3,47 & - & 737,5 & - & - \\
\hline$S_{20}$ & - & - & 0,49 & 2,32 & - & 1736,67 & - & - \\
\hline$S_{21}$ & $-2,17$ & 12,06 & 2,47 & 3,18 & - & 1843,33 & - & - \\
\hline$S_{22}$ & $-0,49$ & 10,92 & 2,84 & 3,18 & $-1,46$ & 746,67 & 1,53 & 0,09 \\
\hline$S_{23}$ & - & - & 0,43 & 2,12 & - & 246,67 & - & - \\
\hline$S_{24}$ & $-2,32$ & 13,02 & 2,56 & 3,47 & - & 2030 & - & - \\
\hline$S_{25}$ & - & - & $-0,38$ & 2 & - & 433,33 & - & - \\
\hline$S_{26}$ & - & - & 0,69 & 2,06 & - & 1840 & - & - \\
\hline$S_{27}$ & $-0,32$ & 10,78 & 2,69 & 3,32 & $-1,85$ & 693,33 & 1,54 & 0,15 \\
\hline$S_{28}$ & $-1,83$ & 8,17 & 2,51 & 3,18 & - & 1271,67 & - & - \\
\hline$S_{29}$ & - & - & 1,76 & 2,43 & - & 915 & - & - \\
\hline$S_{30}$ & $-1,32$ & 11,3 & 2,47 & 3,06 & - & 1146,67 & - & - \\
\hline $\mathbf{S}_{31}$ & $-1,49$ & 11,6 & 2,47 & 3,06 & - & 1260 & - & - \\
\hline$S_{32}$ & $-0,26$ & 10,65 & 2,64 & 3,18 & $-1,49$ & 670 & 1,43 & 0,12 \\
\hline$S_{33}$ & $-0,2$ & 10,92 & 2,05 & 3,12 & $-1,14$ & 697 & 1,21 & $-0,03$ \\
\hline
\end{tabular}

$\Phi=$ représente le diamètre des particules. Moy = Moyenne 
Tableau 2 : Proportion granulométrique des différents groupes.

\begin{tabular}{lcccc}
\hline \multicolumn{5}{c}{ Proportions (\%) } \\
\hline Diamètre $(\boldsymbol{\mu m})$ & Groupe 1 & Groupe 2 & Groupe 3 & \\
\hline 5000 & 5,532 & 46,202727 & 16,8275 & \\
3150 & 10,436 & 69,648182 & 35,25375 & \\
2000 & 13,61 & 75,622726 & 44,84 & sables grossiers \\
1250 & 18,686667 & 79,236364 & 53,29125 & \\
800 & 41,176666 & 82,503637 & 60,429999 & \\
630 & 51,826 & 84,750909 & 65,343749 & \\
\hline 500 & 58,502 & 87,121819 & 70,59125 & \\
400 & 63,145333 & 89,192727 & 75,623751 & sables moyens \\
315 & 68,296667 & 91,678181 & 81,615 & \\
\hline 250 & 74,186667 & 94,236365 & 88,061251 & \\
200 & 79,285334 & 96,030909 & 92,326248 & sables fins \\
160 & 84,581332 & 97,482726 & 95,2075 & \\
125 & 89,251332 & 98,468183 & 97,329999 & \\
\hline 100 & 95,485999 & 99,420002 & 99,01375 & \\
63 & 99,063334 & 99,822726 & 99,545 & sables très fins \\
50 & 99,470001 & 99,847273 & 99,64375 & \\
\hline
\end{tabular}

Tableau 3: Modèles de régression ajustés par saison.

\begin{tabular}{lccccc}
\hline Période & $\begin{array}{c}\text { Nombre de } \\
\text { points }\end{array}$ & Coef. b & Coef. a & Coef. Cor. & Relation \\
\hline Petite saison sèche & 30 & 0,7862 & 0,0205 & 0,80 & $Q_{s}=0,0205 Q_{l}^{0,786}$ \\
Grande saison sèche & 29 & 0,9253 & 0,0117 & 0,88 & $Q_{s}=0,0117 Q_{l}^{0,925}$ \\
Petite saison pluvieuse & 30 & 0,9766 & 0,0054 & 0,64 & $Q_{s}=0,0054 Q_{l}^{0,976}$ \\
Grande saison pluvieuse & 30 & 0,9074 & 0,0051 & 0,91 & $Q_{s}=0,0051 Q_{l}^{0,907}$ \\
\hline \multicolumn{1}{c}{ Ql: débits liquides $\left(\mathrm{m}^{3} / \mathrm{s}\right) ; Q s:$ débits solides en suspension $(\mathrm{kg} / \mathrm{s}) ;$ Coef : coefficient ; Coef. Cor. : Coefficient de corrélation }
\end{tabular}

Tableau 4 : Flux des sédiments en suspension dans le lac d'Ayamé.

\begin{tabular}{llll}
\hline & Crue & Etiage & Moyenne \\
\hline Débit entrant $\left(\mathrm{m}^{3} / \mathrm{s}\right)$ & 87,49 & 28,73 & 58,11 \\
Concentration en MES (mg/L) & 7,7 & 4,6 & 6,15 \\
Flux particulaires entrants (t/j) & 58,20 & 11,41 & 34,80 \\
\hline \multicolumn{1}{c}{ MES = Matières en suspension } & & &
\end{tabular}



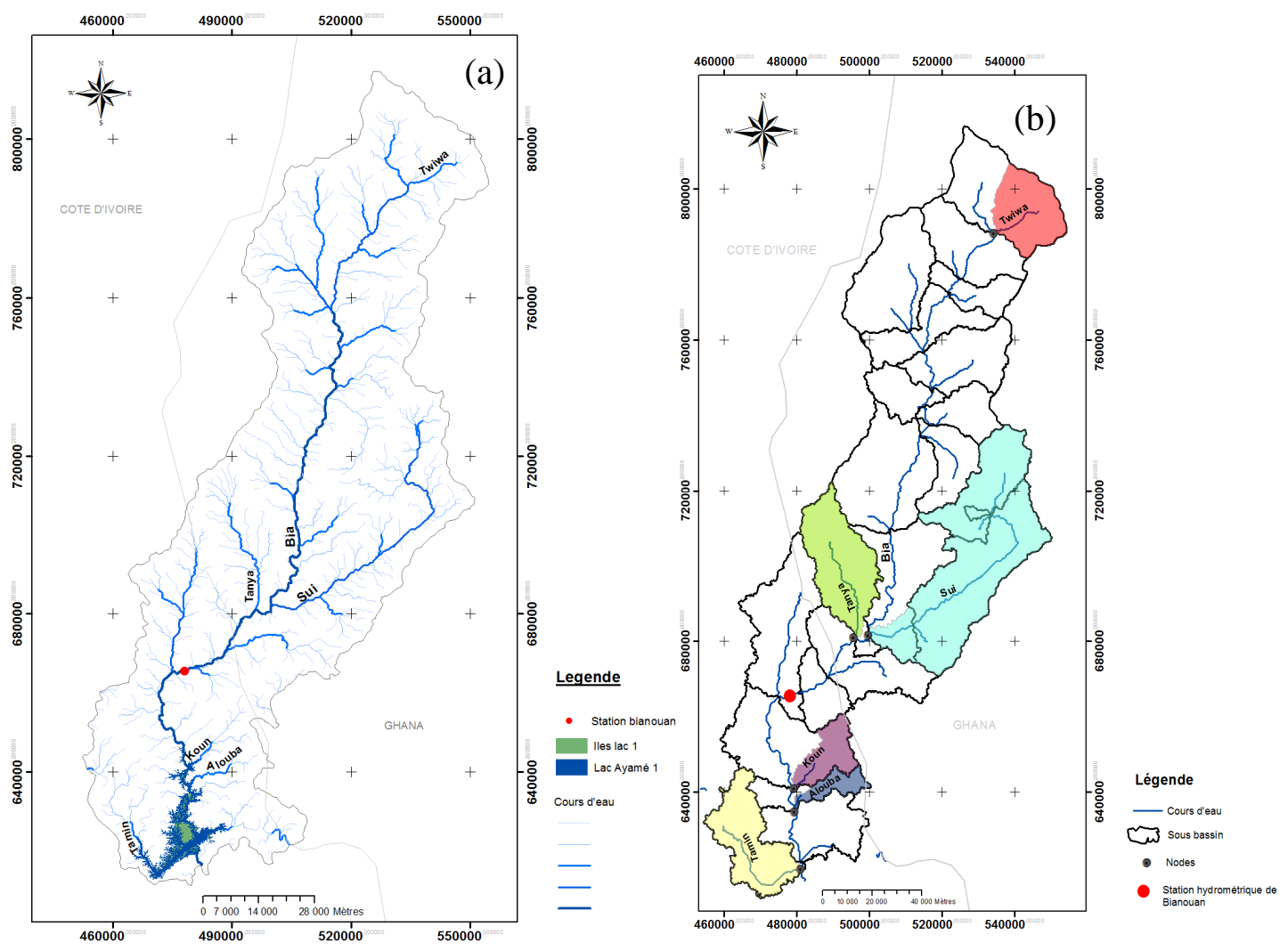

Figure 1: Bassin de la Bia : (a) carte hydrographique ; (b) carte des sous bassins.

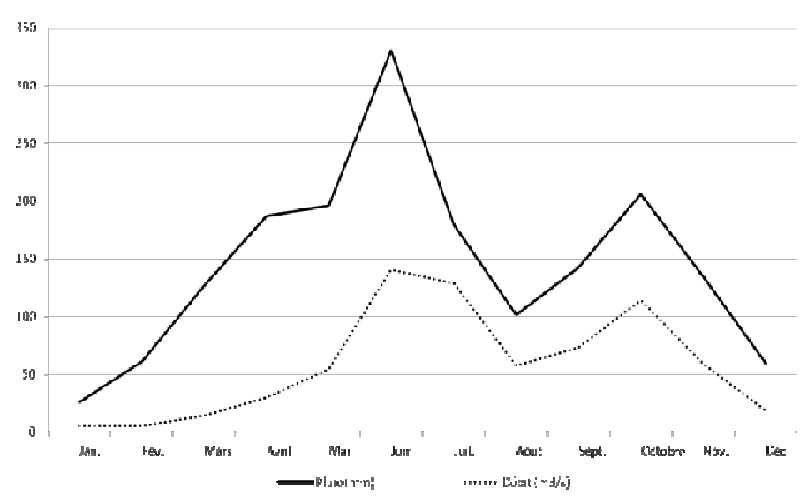

a)

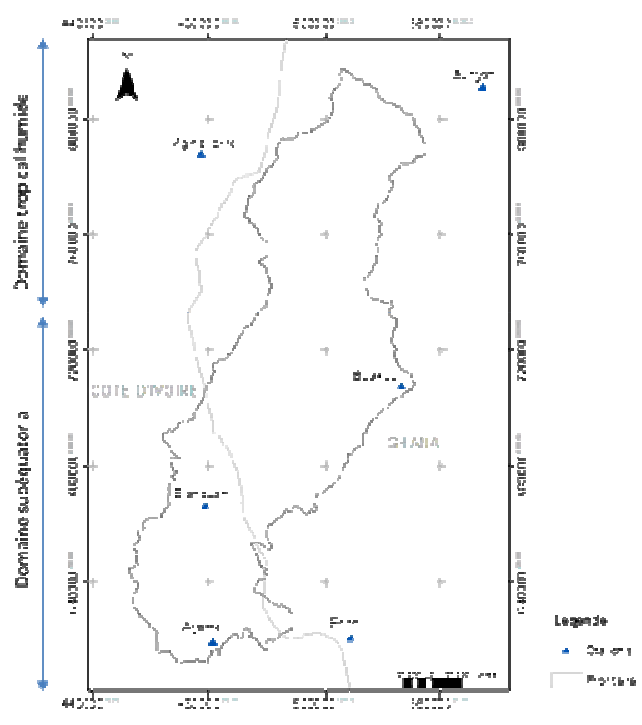

b)

Figure 2 : Climat: a) Variation moyenne mensuelle hydropluviométrique à Ayamé 1 de 2000 à 2013 ; b) Régime pluviométrique du bassin de la Bia. 


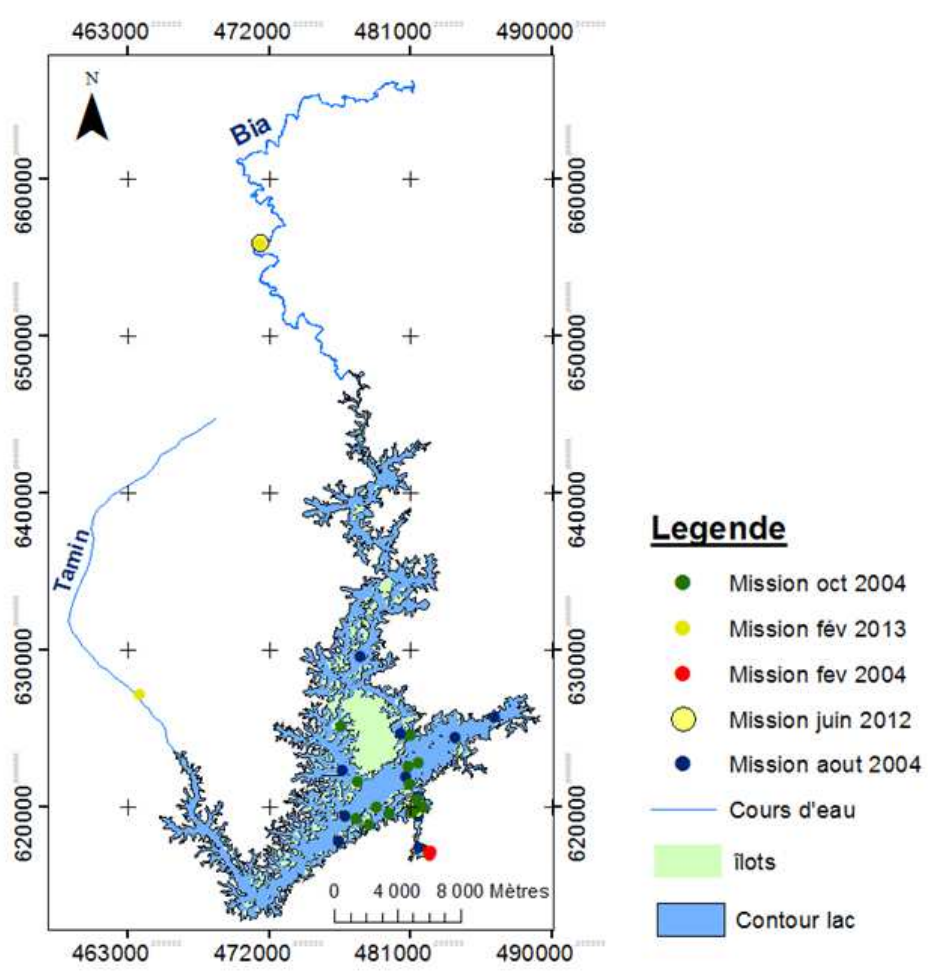

Figure 3: Points de prélèvement des échantillons.

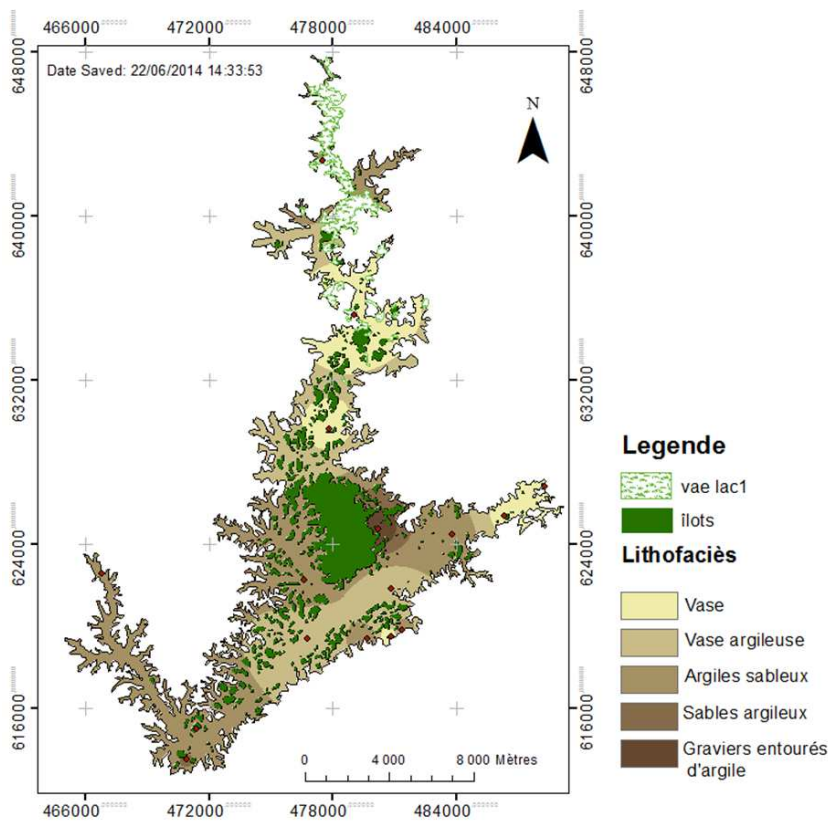

Figure 4: Lithofaciès du lac d'Ayamé 1. 


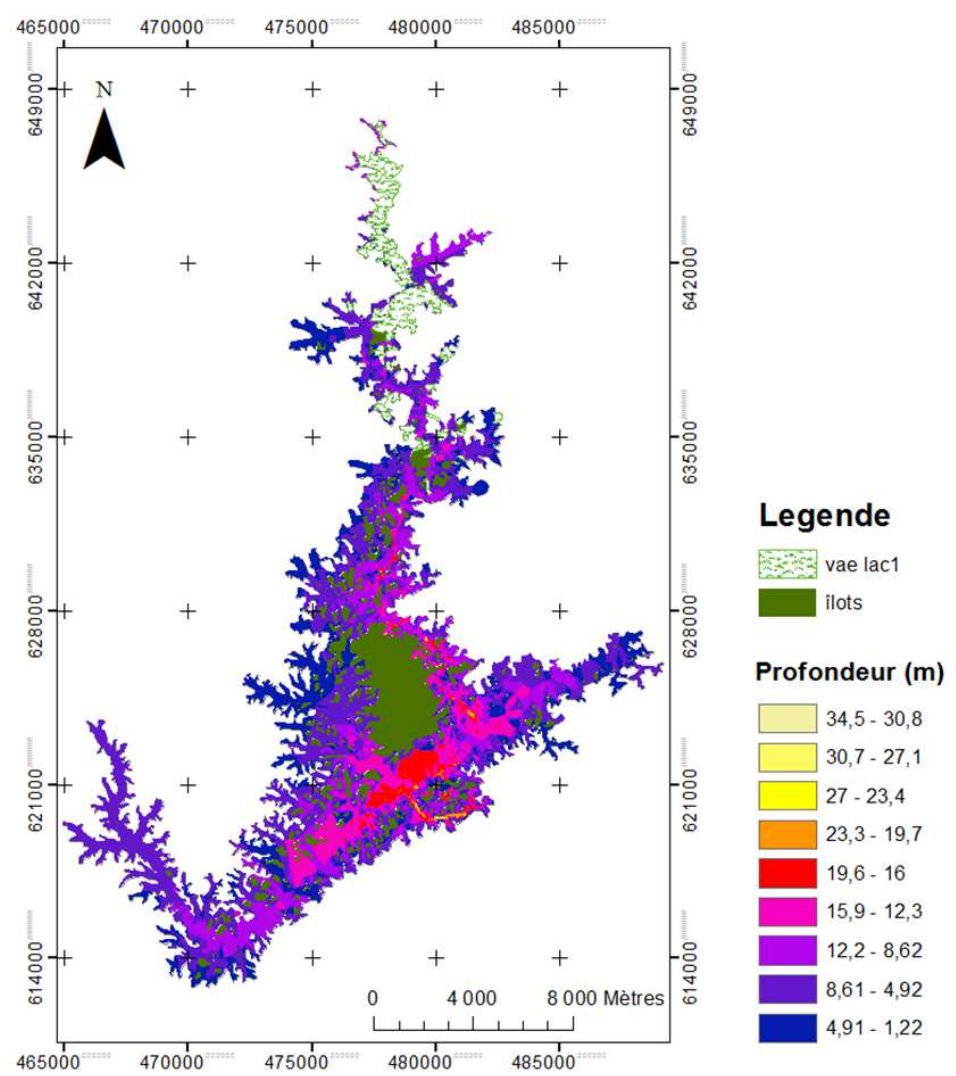

Figure 5: Bathymétrie du lac d'Ayamé 1en 2005.

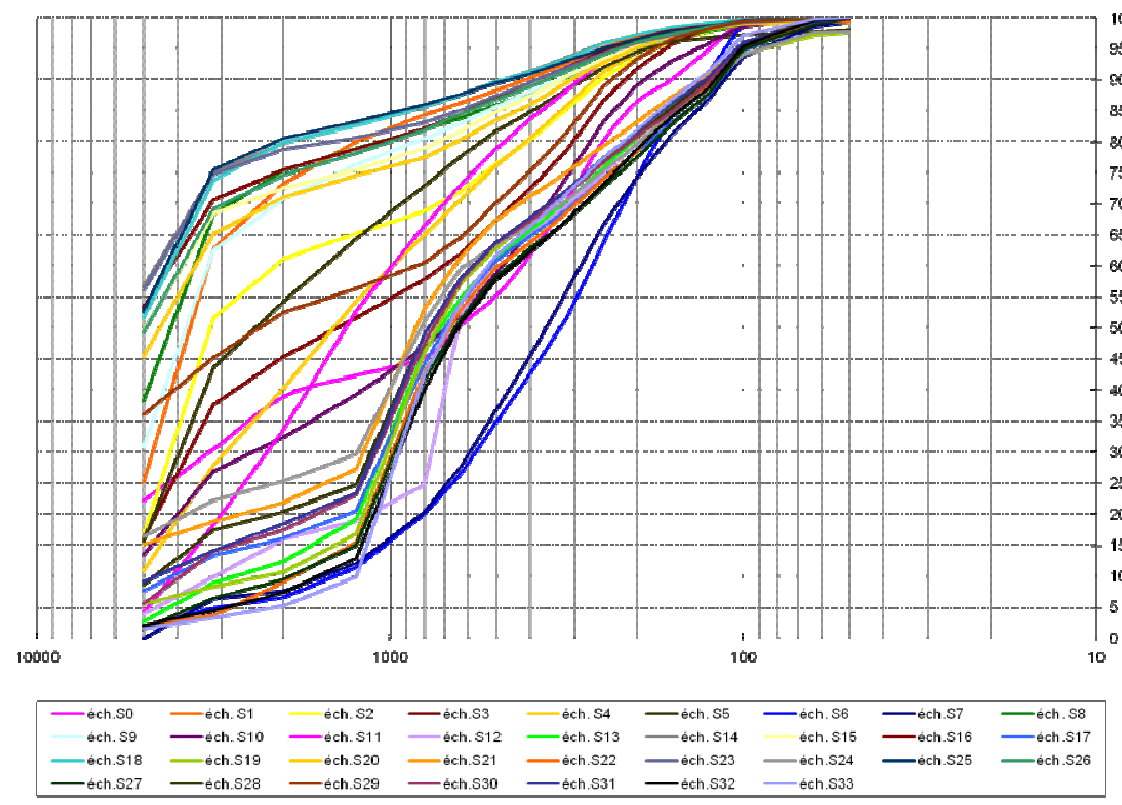

Figure 6 : Courbes cumulatives des sédiments. 


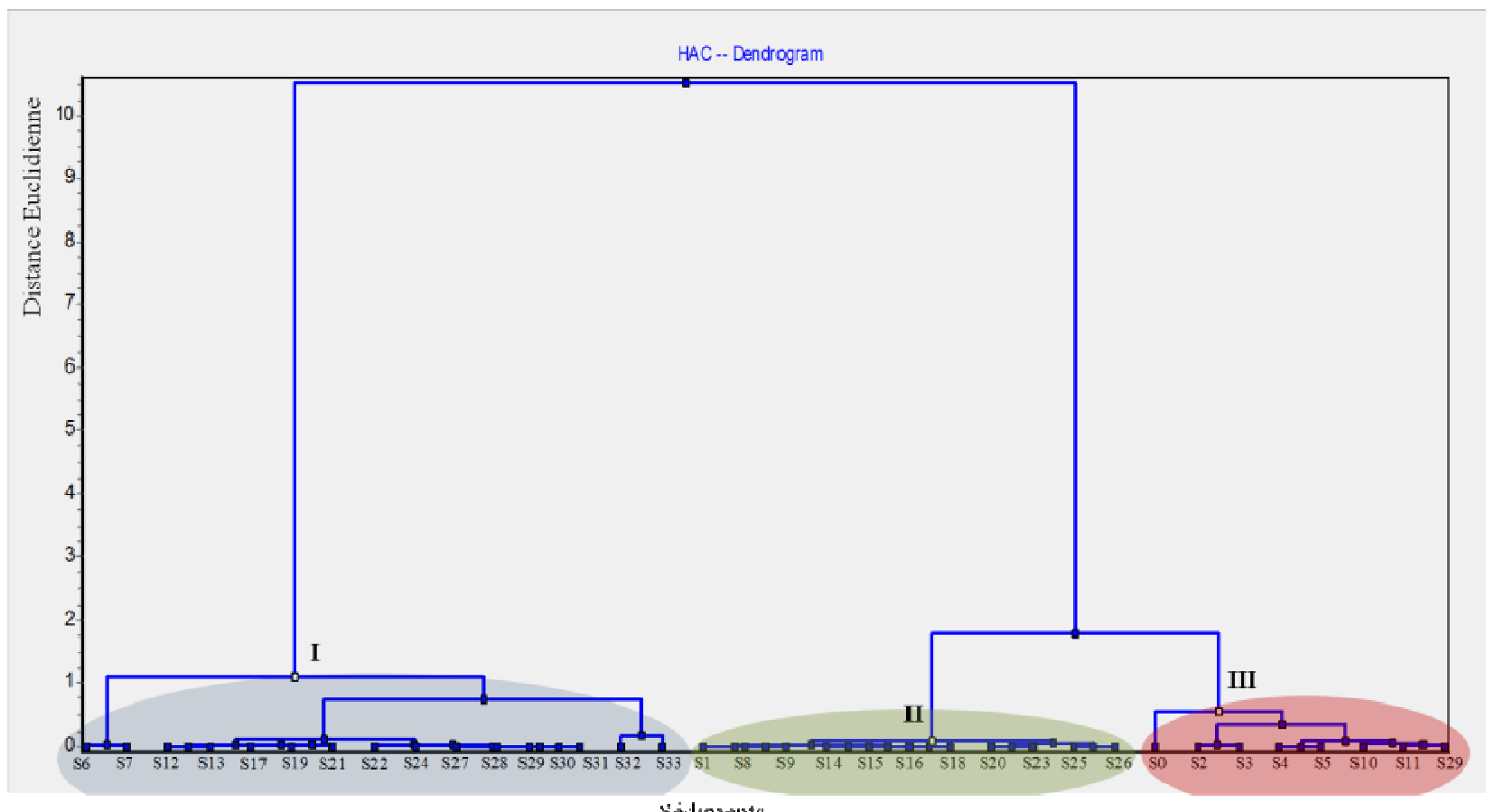

Figure 7 : Dendrogramme de classification des sédiments du lac d'Ayamé par rapport à la taille des grains. 


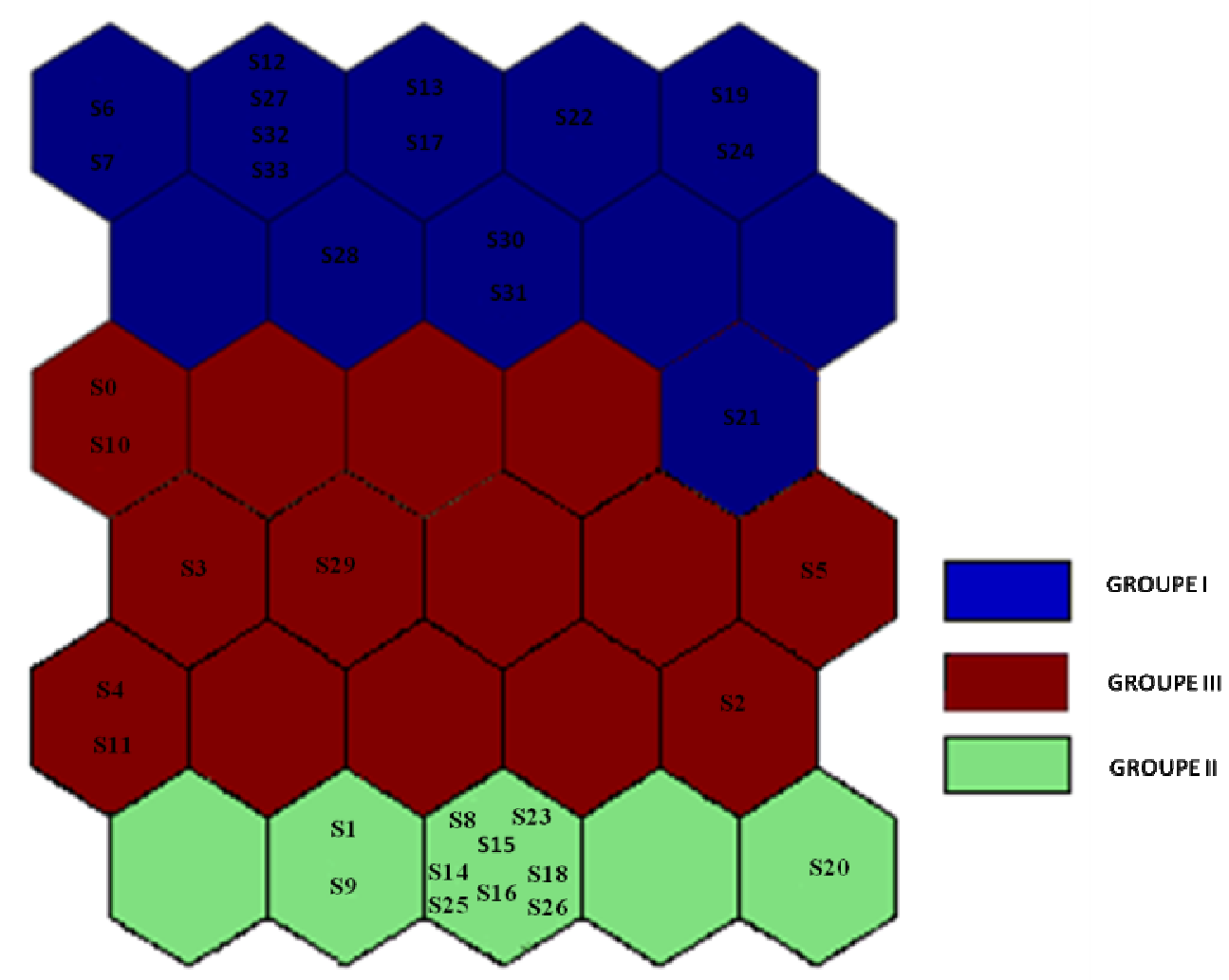

Figure 8 : Distribution des échantillons de sédiments en fonction de leur similitude sur la carte de Kohonen. 
N. H. MELEDJE et al. / Int. J. Biol. Chem. Sci. 8(3): 1290-1307, 2014
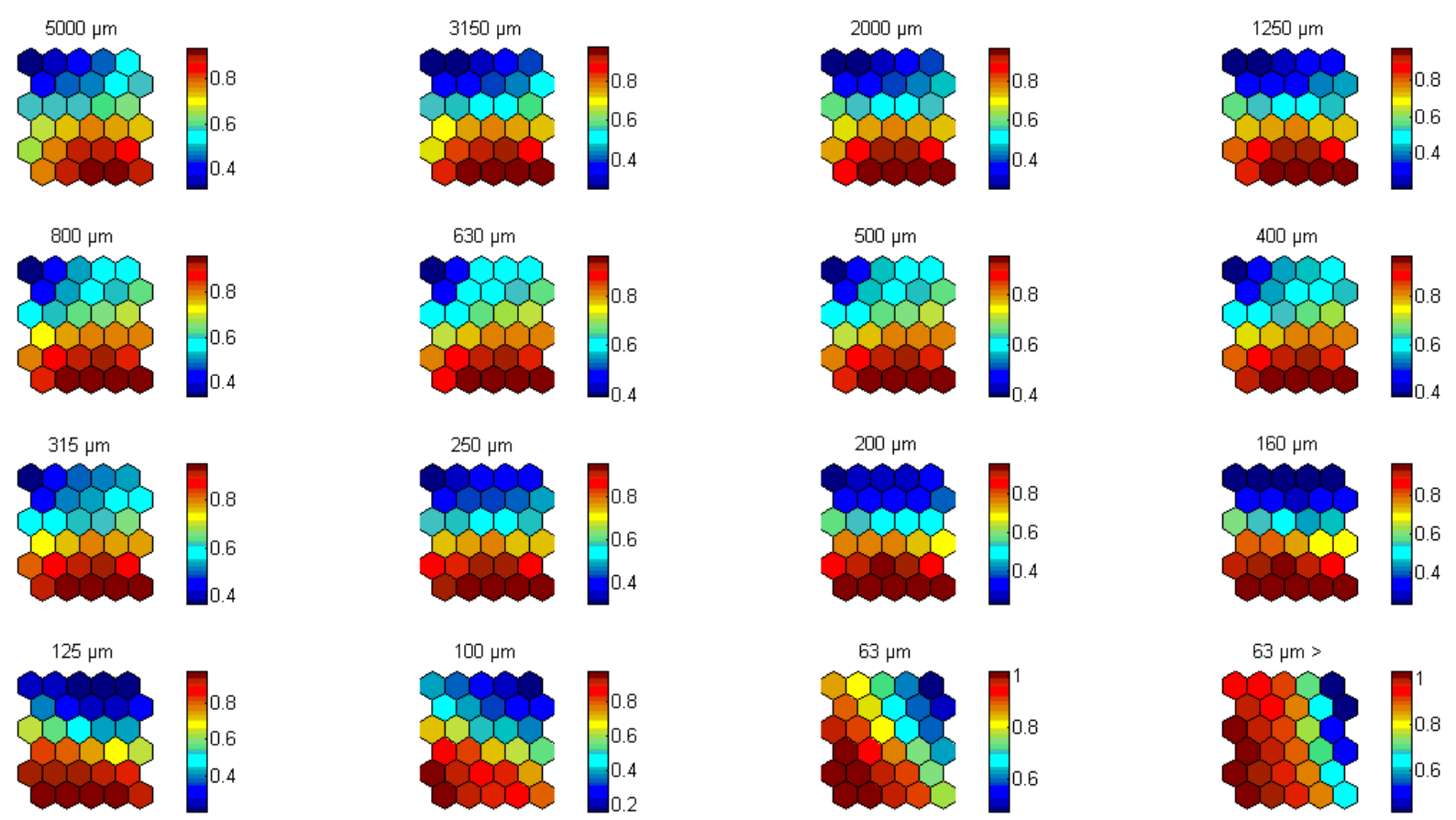

Figure 9 : Gradient de valeurs de chaque diamètre granulométrique sur la carte de Kohonen. 


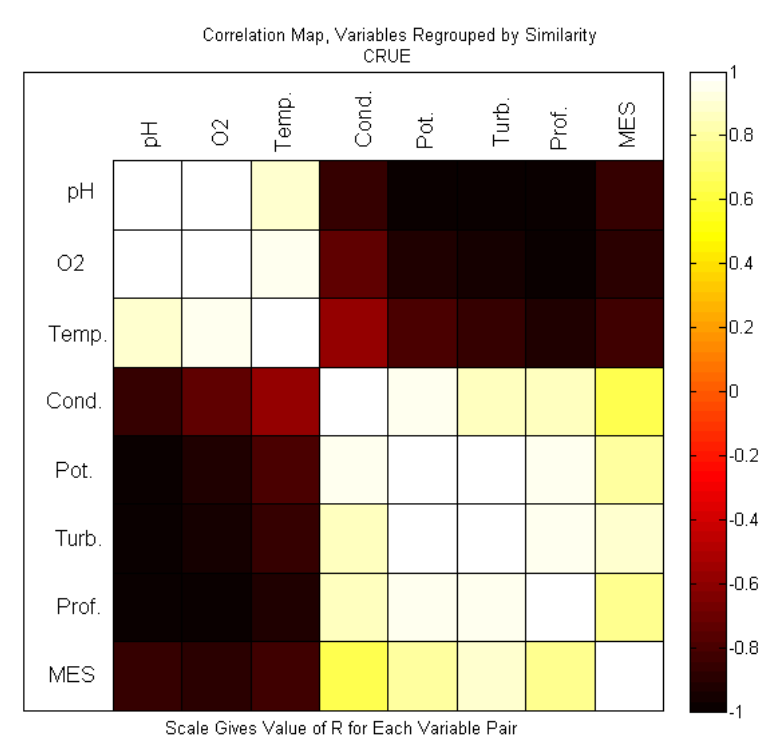

(a)

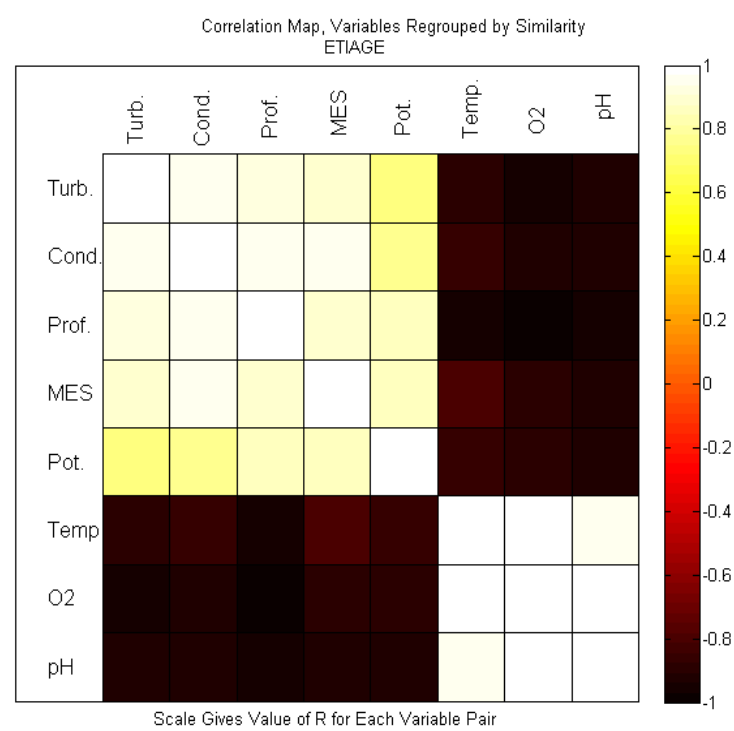

(b)

Figure 10: Matrice de corrélation des caractéristiques physicochimiques des eaux du lac d'Ayamé 1 ; a) : pendant la saison des pluies ; b) : pendant la saison sèche. Turb= Turbidité $;$ Temp= Température $;$ Cond= conductivité $;$ Pot= potentialité $;$ MES= Matières en suspension; Prof= Profondeur; O2=Oxygène dissous 


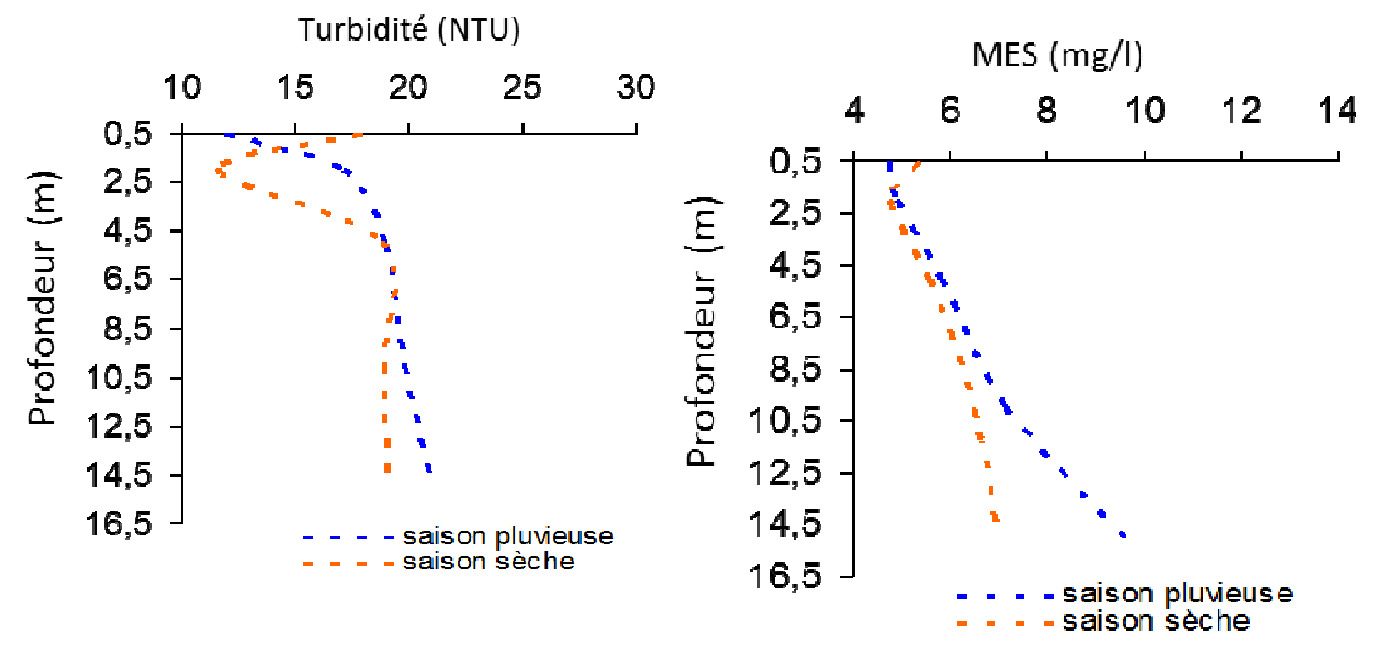

Figure 11 : Evolution saisonnière des charges solides en suspension du lac d'Ayamé.
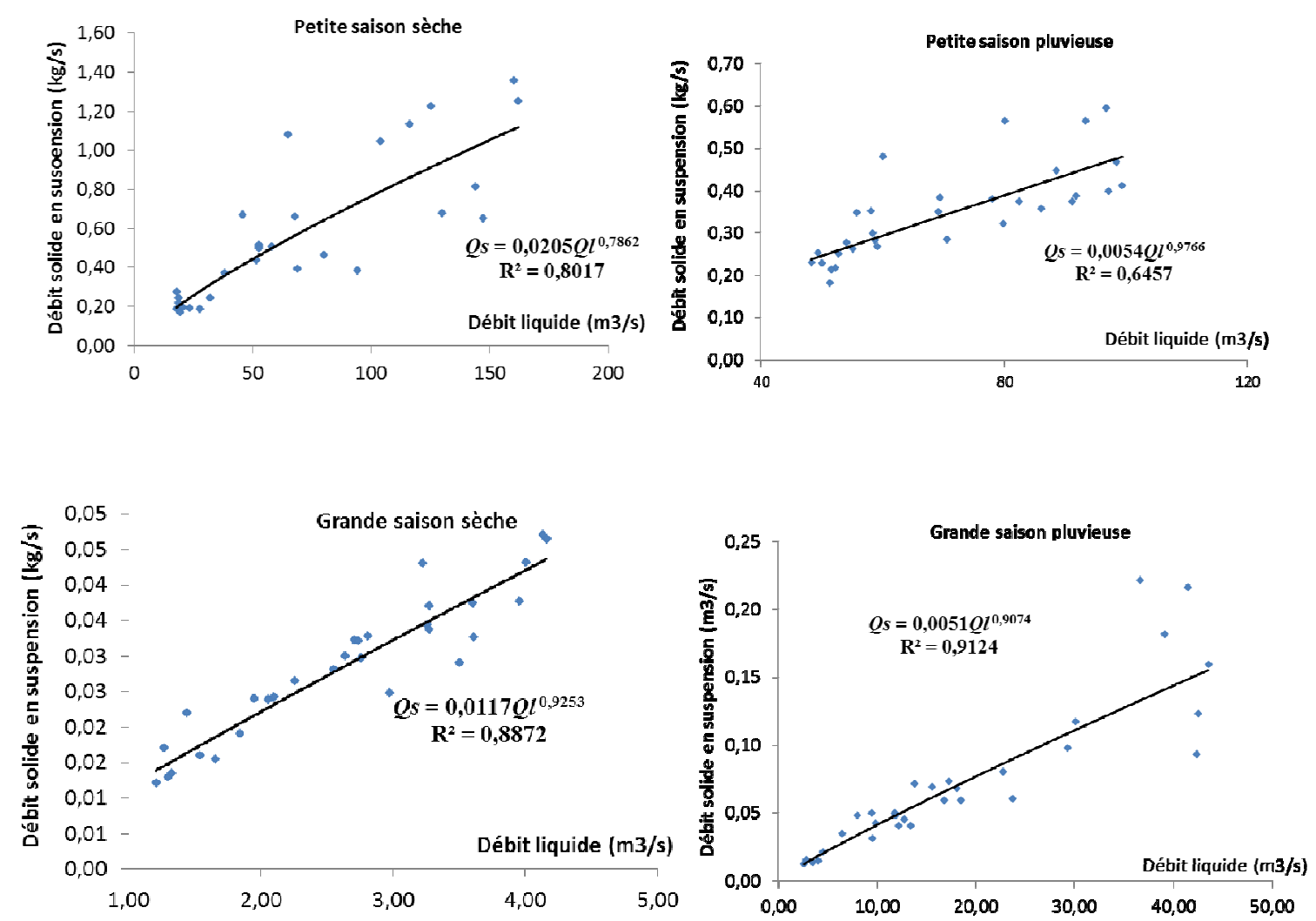

Figure 12 : Corrélation entre débit solide et débit liquide à différentes saisons. 


\section{DISCUSSION}

L'analyse des paramètres physicochimiques du lac d'Ayamé 1 montre que la turbidité a une variation similaire avec les concentrations des MES. Ils présentent en général un gradient vertical positif dans la colonne d'eau aussi bien en saisons sèches qu'en saisons pluvieuses. L'augmentation progressive des valeurs de la turbidité avec la profondeur serait sans doute en liaison avec la présence des MES qui, par décantation, s'accumulent vers le fond du plan d'eau. La présence de particules en suspension et de matières colloïdales peut affecter la transparence de l'eau. La turbidité étant ellemême le paramètre qui détermine la transparence de l'eau. Les valeurs moyennes de turbidité à Ayamé 1 (17,50 NTU) indiquent que les eaux sont légèrement troubles selon Alayat (2014) qui souligne dans sa classification que la turbidité du lac Oubeira en Algérie comprise entre 5 NTU et 30 NTU est légèrement trouble. A Ayamé 1, les eaux sont plus chargées en saison pluvieuse $(7,70 \mathrm{mg} / \mathrm{L}$ en moyenne) qu'en saison sèche $(4,6 \mathrm{mg} / \mathrm{L}$ en moyenne). Cette augmentation des charges solides en suspension en saison pluvieuse serait liée aux apports des eaux de ruissellement, assez chargées en sédiment, dans le lac. Bouanani (2004) rapporte que l'importance et la nature du débit solide en suspension sont liées d'une part à l'intensité de l'érosion par l'eau de ruissellement; donc à l'importance des précipitations et, d'autre part, à la capacité de l'écoulement à transporter la matière solide. Les charges solides en suspension dans le lac d'Ayamé 1 sont relativement faibles par rapport à la charge solide obtenue par Kouassi (2007) et Kouassi et al. (2013) dans le lac de Taabo (12 mg/L).

Cette différence pourrait s'expliquer par le fait que le bassin du lac de Taabo se trouvant dans une région de savane à un sol beaucoup plus exposé à l'érosion par rapport à la région d'Ayamé qui se trouve dans une zone forestière. Les résultats des modèles d'ajustement: débit solide - débit liquide sont assez significatifs, puisque le coefficient de corrélation varie entre 0,64 et 0,91 pour toutes les applications. En ce qui concerne la relation liant les débits liquides aux débits solides en suspension qui est de type puissance de forme $Q_{g}=a Q_{l}^{b}$, le coefficient de corrélation est beaucoup plus important pour la grande saison $(0,91)$. Ceci peut s'expliquer par la régularité des apports durant cette saison. Le modèle saisonnier montre que les paramètres $a$ et $b$ sont différents de ceux trouvés par bon nombre de chercheurs. Les valeurs de l'exposant b de la région d'Ayamé est comprises entre 0 et 1 alors que celles trouvées à Oued Mouilah et Oued Sebdou (Bouanani, 2004), à Oued Mina (Touaibia et al., 2001), à Oued Wahrane (Benkhaled et Remini, 2003) sont comprises entre 1 et 2. Ceci pourrait s'expliquer par le fait que le transport solide en suspension dans le lac d'Ayamé est faible par rapport aux valeurs obtenues par ces mêmes auteurs. Le cours d'eau apporte par saison en moyenne 58,11 $\left(\mathrm{m}^{3} / \mathrm{s}\right)$ d'eau. Ces quantités d'eau apportent journalièrement 34,80 tonnes de sédiments fins en suspension.

\section{Conclusion}

Les études effectuées dans le lac du barrage hydroélectrique d'Ayamé 1, au cours de nos campagnes réalisées en périodes de crue (juin et octobre 2004, juin et octobre 2012, juin et octobre 2013) et en périodes d'étiage (février et août 2004, février et août 2012, février et août 2013), nous ont montré que le fond du lac est très vaseux. Les flux sédimentaires venant du ravinement des berges et des eaux de la Bia couplés à la forte activité anthropique, concourent au comblement des fonds. Sur la base des mesures réalisées, nous avons calculé les différentes valeurs des paramètres de l'écoulement liquide et du transport solide. On peut dire que le lac d'Ayamé semble être le principal réceptacle sédimentogène, recevant une partie des matériaux transportés par la Bia. Ainsi, pendant les périodes de crue, les charges solides sont relativement plus élevées (7,7 mg. $\left.\mathrm{L}^{-1}\right)$ que pendant les périodes d'étiage $\left(4,6 \mathrm{mg} \cdot \mathrm{L}^{-1}\right)$. Les charges solides en 
suspension ont une valeur moyenne de 6,15 mg. $\mathrm{L}^{-1}$.

Les charges solides sont plus importantes en saison pluvieuse qu'en saison sèche. Concernant les relations statistiques de forme $Q_{z}=a Q_{l}^{b}$, les analyses mettent en évidence que le modèle puissance peut être utilisé pour obtenir des relations acceptables. Les analyses granulométrique ont montré que les sédiments rencontrés dans le fond du lac d'Ayamé 1 sont constitués de vases, de vase argileuse, argiles sableux et de graviers. La classification a permis de regrouper les sédiments en 3 classes ayant les caractéristiques semblables à partir de leur diamètre. Le groupe I est caractérisé par un faible taux $(5,532 \%)$ de sédiments de diamètre $5000 \mu \mathrm{m}$. le groupe II renferme comprend majoritairement des sédiments grossiers $(84,75 \%)$. Les sédiments de ce groupe sont les plus riches en éléments grossiers et fins. Le troisième groupe se caractérise par des sédiments grossiers $(65,34 \%)$ à moyen $(16,27 \%)$. Cette étude a également permis de réaliser la première carte bathymétrique du lac d'Ayamé 54 ans après sa mise en eau. Les résultats devraient permettre d'estimer, à terme, la durée de vie du barrage hydroélectrique d'Ayamé 1.

\section{REFERENCES}

Adjanohoun E, Guillaumet JL. 1971. La végétation. In Le Milieu Naturel de Côte d'Ivoire. Mém. ORSTOM Paris, 50: 161162.

Alayat H, Kherici N, Lamouroux C. 2014. Space evolution of the silting of Oubeira lake imposed by erosion (extreme ne algerian). Revue LJEE, 15: 28-38.

Bamory Kamagate, Adama Mariko, Luc Seguis, Amidou Dao, Hamadou Bokar, Droh Laciné G. 2010. Differenciation hydrogeochimique entre les nappes superficielles des altérites et profondes du socle fissuré dans le bassin versant de Kolondièba (Sud du Mali): approche statistique par la méthode SOM des réseaux de neurones. IAHS Publ., 340.
Benkhaled A, Remini B. 2003. Variabilité temporelle de la concentration en sédiments et phénomène d'hystérésis dans le bassin de l'Oued Wahrane (Algérie). Journal des Sciences Hydrologiques, 48(2) : 243-255.

Bessenassé M, Kettab A, Paquier A, Ramez P, Galea G. 2003. Simulation numérique de la sédimentation dans les retenues de barrages : cas de Zardezas, Algérie. Sci. Eau, 16 : 103-122.

Bouanani A. 2004. Hydrologie, transport solide et modélisation. Etude de quelques sous bassins de la Tafna (NW Algérie). Thèse de Doctorat d'Etat, Université Abou Bekr Belkaid, Tlemcen, Algérie.

Christian FL, Kenneth NB, Daniel Heneley, Joseph AM. 2010. Spatial and temporal variation in suspended sediment, organic matter, and turbidity in a Minnesota prairie river: implications for TMDLs. Environmental Monitoring and Assessment, 165: 435-447.

Durand J, Guiral D. 1994. Hydroclimat et hydrochimie. Environnement et ressource aquatique de Côte d'Ivoire. Tome 2 Les milieux lagunaires (Durand J, Dufour P, Guiral D, Zabi S. Mém. ORSTOM, 50: 129-136.

Folk R, Word W. 1957. Brazors river bors, a study in significance of grain size parameters. J. Sedim. Petrol., 27: 13-27.

Girard G, Sircoulon J, Touchehbeuf P. 1970. Aperçu sur les régimes hydrologiques de Côte d'Ivoire. Orstom Centre d'Adiopodoumé, Service Central Hydrologique, Rapport ORSTOM, p.91.

Grismer ME. 2012. Erosion modelling for land management in the Tahoe basin, USA:scaling from plots to forest catchments. Journal des Sciences Hydrologiques, 57(5): 878-899.

Groga N. 2012. Structure, fonctionnement et dynamique du phytoplancton dans le lac de Taabo (Côte d'Ivoire). Thèse de doctorat, Université de Toulouse, p.224.

Kaki CA, Agbani KMA. 2000. Le domaine margino- littoral Sud-Ouest Béninois: Evolution récente du milieu sédimentaire. 
Actes des Journées Scientifiques Internationales de l'UNB, 215-229.

Kouassi KL. 2007. Hydrologie, transport solide et modélisation de la sédimentation dans les lacs des barrages hydroélectriques de Côte d'Ivoire: Cas du lac de Taabo. Thèse de Doctorat, Université d'Abobo-Adjamé, p.209.

Kouassi KL, Kouamé KI, Konan KS, Angoulo MS, Demé M, Meledje NH . 2013. TwoDimensional Numerical Simulation of the Hydro-Sedimentary Phenomena in Lake Taabo, Côte d'Ivoire. Water Resources Management, 27(12): 4379-4394.

Kouassi L, Kouamé I, Konan S, Dibi B, Moussa S, Koné D, Gnakri D. 2013. Prospects for Minimizing the Potential Environmental Impacts of the HydroAgricultural Dam of M'Bahiakro (Côte d'Ivoire). Journal of Water Resource and Protection, 5: 847-853.

Kohonen T. 2001. Self-Organizing Maps (3e edn). Springer-Verlag : Berlin, Germany; $362 p$.

Lenhart CF, Brooks KN, Heneley D, Magner JA. 2010. Spatial and temporal variation in suspended sediment, organic matter, and turbidity in a Minnesota prairie river: implications for TMDLs. Environ Monit Assess, 165: 435-447.

Kouamé MK, Dietoa MY, Edia EO, Da Costa SK, Ouattara A, Gourène G. 2011. Macroinvertebrate communities associated with macrophyte habitats in a tropical man-made lake (Lake Taabo, Côte d'Ivoire). Knowledge and Management of Aquatic Ecosystems, 400, 03; http://www.kmae-journal.org.

Kouamé MK, Diétoa MY, Da Costa SK, Edia EO, Ouattara A and Gourène G. 2010. Aquatic macroinvertebrate assemblages associated with root masses of water hyacinths, Eichhornia crassipes (Mart.) Solms-Laubach, 1883 (Commelinales: Pontederiaceae) in Taabo Lake, Ivory Coast. Journal of Natural History, 44(58): 257-278.

N'go YA. 2000. Etude de l'érosion des sols de la région de Buyo: analyse des facteurs et essai d'évaluation des risques par la télédétection et les systèmes d'information géographique. Thèse de $3^{\text {ème }}$ cycle, Université d'Abobo-Adjamé, p. 155 .

Pothin KBK, Assié EK. 2000. Etude du contexte lithostratigraphique et de la minéralisation aurifère de la région de l'Aféma (Sud-Est de la Côte d'Ivoire) à partir des échantillons de sondages carottés. Bioterre, Rev. Inter. Sci. de la Vie et de la Terre, 1: 22-35.

Okacha T, Abdelkader M, Med AT. 2002. Mise en place d'un système d'information géographique pour le suivi et la quantification de l'érosion hydrique: application au bassin versant de l'Isser (Tlemcen). Sécheresse, 13: 175-179.

Ouattara A. 2000. Premières données systématiques et écologiques du phytoplancton du lac d'Ayamé (Côte d'Ivoire). Thèse de Doctorat Unique, Universiteit Katholieke de Louvain, Belgique, p.207.

Ryan A. Harnish, Rishi Sharma, Geoffrey A. McMichael, Russell B. Langshaw, Todd NP. 2014. Effect of hydroelectric dam operations on the freshwater productivity of a Columbia River fall Chinook salmon population. Can. J. Fish. Aquat. Sci., 71: 602: 615.

Saaidi E. 1991. Traité de Sédimentologie. Ellipses Edition : Paris; 393.

Touaibia B, Gomer D, Aidaoui A, Achite M. 2001. Quantification et variabilité temporelles de l'écoulement solide en zone semi-aride, de l'Algérie du Nord. Hydrol. Sci. J., 46(1): 41-53.

Yapo OB. 2002. Evaluation de l'état trophique du lac de Buyo (Côte d'Ivoire). Thèse de Doctorat Unique, Université d'Abobo-Adjamé, Côte d'Ivoire, p.296.

Zimmerman J, Westra J, Vondracek B. 2003. Agricultural land use effects on sediment loadingand fish assemblages in two Minnesota (USA) watersheds. Environmental Management, 32(1): 93105. 